\title{
RHODOCOCCUS EQUI BACTEREMIA WITH LUNG ABSCESS MISDIAGNOSED AS CORYNEBACTERIUM. A REPORT OF 2 CASES
}

Felipe Francisco Tuon, Rinaldo Focaccia Siciliano, Tarik Al-Musawi, Flavia Rossi, Vera Luiza Capelozzi, Ronaldo Cesar Gryschek, Eduardo Alexandrino Sérvolo Medeiros

\section{INTRODUCTION}

Rhodococcus equi infection is commonly encountered in HIV-infected patients; recipients of organ transplants; and in those with lymphoma, chronic renal failure, alcoholism, lung cancer, leukaemia, diabetes mellitus, and other states of immunodeficiency. Recent reports have also documented $R$. equi infections among immunocompetent patients. ${ }^{1}$ The most common manifestations of $R$. equi infections are multiple abscesses in the lungs, and extrapulmonary infections may include wound infections, subcutaneous abscesses, brain abscesses, meningitis, pericarditis, osteomyelitis, cervical adenopathy, endophthalmitis, lymphangitis, or mastoiditis. ${ }^{2}$ We report 2 cases of lung abscesses with $R$. equi misdiagnosed as Corynebacterium species.

\section{CASE REPORT}

\section{Case 1}

A 32-year-old man was admitted to the hospital in September of 2005 with 2 days of fever and 6 weeks of weight loss and anorexia. He had a history of Hodgkin lymphoma in 1992 and a secondary non-Hodgkin lymphoma in 2000, with pulmonary Nocardia diagnosed together with the relapse of lymphoma in 2002. He had undergone successful autologous bone marrow transplantation in April of 2004. His physical examination was within normal limits, but $\mathrm{Co}$ rynebacterium sp grew in 3 separate blood cultures. He was observed for 2 days, and the clinician considered the isolated bacteria to be a contaminant. He was discharged to home 5 days after admission when he became afebrile and had a normal examination, including thorax computed to-

Department of Infectious and Parasitic Diseases, Faculdade de Medicina, Universidade de São Paulo, São Paulo Brazil Email: ffbt@pop.com.br mography. After 1 month, he was readmitted to the hospital with right side hemiparesis, nonproductive cough, and fever again. A new chest computed tomography demonstrated multiple nodular infiltrates in both lungs with 2 cavities (Figure 1). A brain magnetic resonance revealed a left temporal nodular lesion with oedema. The patient had to be admitted to the intensive care unit within 24 hours of admission because of a progressive decrease in the level of consciousness; then, dexametasone $24 \mathrm{mg}$ was initiated. Blood cultures were collected, and vancomycin $2000 \mathrm{mg}$ every day was initiated due to prior isolation of Corynebacterium from the blood 1 month previously; ceftriaxone (2000 mg every day) and trimethoprim/sulfamethoxazole (20 mg/kg/day of sulfamethoxazole) were added because of past nocardiosis; rifampin $600 \mathrm{mg}$, isoniazid $400 \mathrm{mg}$, and pyrazinamide $2000 \mathrm{mg}$ were added to cover for the possibility of Mycobacterium tuberculosis infection. Additionally, the patient had hyponatremia and hyperkalemia, and acute adrenal insufficiency was diagnosed by a cosyntropin test.

The results of cultures from bronchoalveolar lavage were negative. The bacteria from the first blood cultures were later identified as Rhodococcus equi. A transesophageal echocardiogram was negative for endocarditis and showed cardiomyopathy with an ejection fraction of $31 \%$. An HIV screening test was negative. Urinalysis was normal. The blood cultures before starting vancomycin were positive for $R$. equi, and after 3 weeks of antibiotic, 2 new blood cultures were still positive. His treatment continued with vancomycin, rifampin, and trimethoprim/sulfamethoxazole, but blood cultures were positive for $R$. equi 6 weeks after admission; the tuberculostatic medications and ceftriaxone were discontinued. Vancomycin serum levels were determined, and the peak and basal levels, $32 \mu \mathrm{g} / \mathrm{mL}$ and $17 \mu \mathrm{g} /$ $\mathrm{mL}$, respectively, were acceptable. An E-test (Progressive Diagnostics Manufacturers epsilometer test; AB Biodisk, Solna, Sweden) of susceptibility to vancomycin was performed with a result of $1 \mu \mathrm{g} / \mathrm{mL}$. A stereotactic biopsy of 


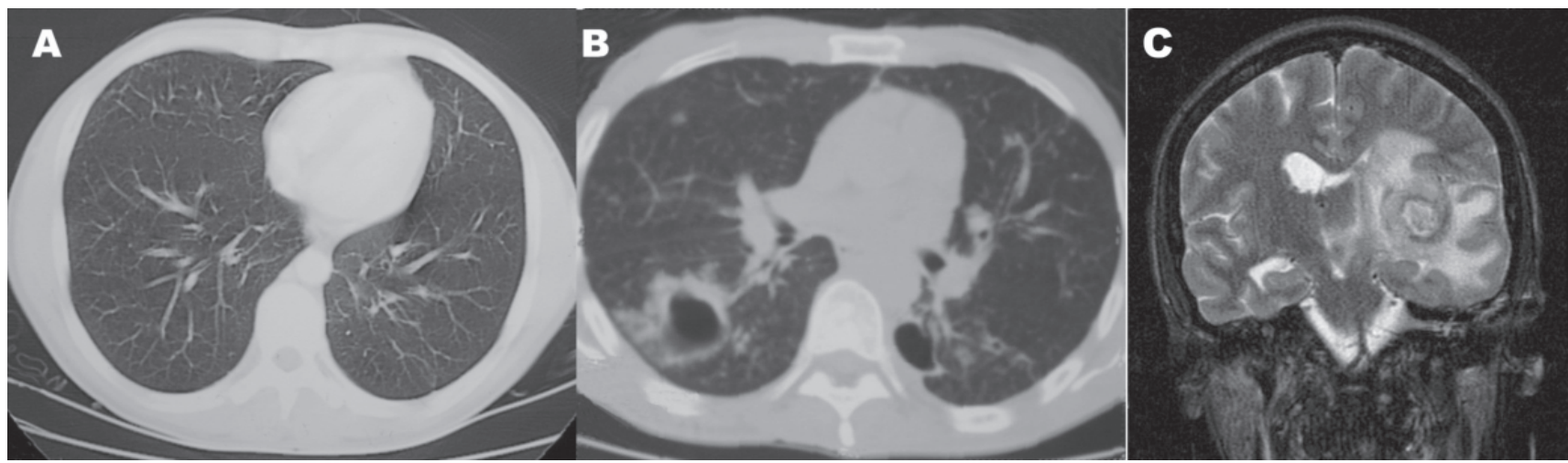

Figure 1 - Thorax CT-scan showing a lung abscess in a patient with Rhodococcus equi bacteremia (B). The normal CT-scan 1 month before symptoms (A). Magnetic resonance with a brain abscess (C).

the brain lesion was inconclusive showing only necrotic material and negative cultures. The patient died after 7 weeks of therapy due to acute respiratory distress and refractory shock.

\section{Case 2}

A 39-year-old woman was admitted in January 2001 with a 2-month history of productive cough, night fever, anorexia, and weight loss. Fine crackles were presented in the right lung, and a discrete hepatomegaly found. An xray showed a cavity in the right lung, which was confirmed by a thorax CT (Figure 2). A HIV test was positive, and the $\mathrm{CD}^{+} \mathrm{T}$ lymphocyte count was $1 \mathrm{cell} / \mathrm{mm}^{3}$. Corynebacterium sp grew in 5 separate blood cultures collected at admission and subsequently in bone marrow and bronchoalveolar lavage cultures. The bacteria from the blood, bone marrow, and bronchoalveolar lavage cultures were later identified as $R$. equi, and intravenous imipenem $2000 \mathrm{mg}$ every day associated with oral rifampin $1200 \mathrm{mg}$ every day was initiated. Blood cultures were negative after 8 days of antibiotic therapy. A right lung pneumectomy was performed after 40 days due to absence of radiological improvement and maintenance of a low-grade fever and productive cough. A pleural fistula was diagnosed 21 days postsurgery, and the patient died 3 days later with septic shock. A necropsy was performed, and sections of the inflated and formalin-fixed right lung revealed a yellow solid and irregular mass in the middle lobe. Histologically, the mass was represented by sheets of macrophages with foamy or abundant granular eosinophilic cytoplasm that contained Michaelis-Gutmann bodies, characterized by visible PASpositive and diastase-resistant basophilic structures with targetoid-like concentric laminations. Gram-stained sections were negative for bacteria, as were stains for fungi and acidfast organisms.

\section{DISCUSSION}

Rhodococcus equi is a bacterium identified in a variety of soils, water, and animals, including horses, cattle, swine, and wild birds. ${ }^{3}$ Rhodococcus equi infection is rare, but the incidence has increased markedly, which is coincident with the pandemia of AIDS, organ transplantation, and

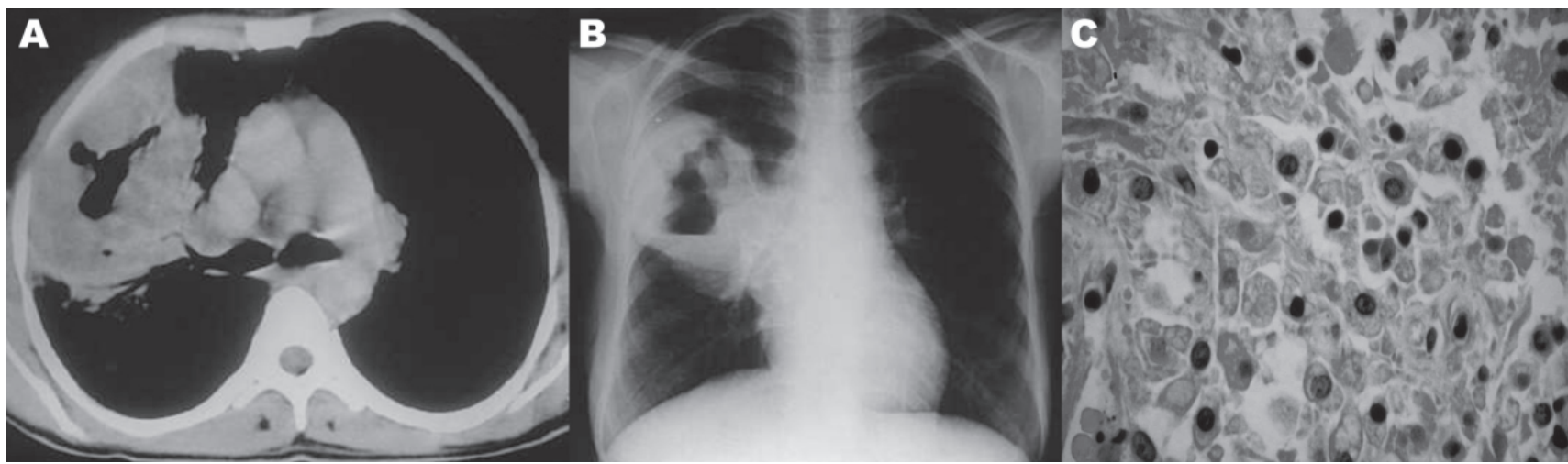

Figure 2 - Thorax CT-scan (A) and Rx-ray (B) of Rhodococcus equi bacteremia with lung abscess. Lung histological section with sheets of macrophages with foamy or abundant granular eosinophilic cytoplasm that contain Michaelis-Gutmann bodies, characterized by visible PAS-positive and diastaseresistant basophilic structures with targetoid-like concentric laminations (C), (HE 400x). 
cancer treatment. Improvements in laboratory techniques and recognition of gram-positive bacilli as pathogens also explain the increase in the incidence of reports of $R$. equi infection. ${ }^{3}$ More than 100 cases of human Rhodococci infections have been published since $1967,{ }^{4}$ when the first $R$. equi infection in a human was reported. ${ }^{5}$ Rhodococci belong to the family Nocardiaceae, which includes Corynebacterium, Mycobacterium, Nocardia, and Gordonia species. It is a nonmotile, gram-positive, obligate aerobe capable of metabolizing a wide variety of organic compounds. On the basis of its morphologic characteristics, $R$. equi can easily be mistaken for a diphtheroid contaminant, such as Corynebacterium or Micrococcus organisms. On the basis of acid-fast staining results, it can be mistaken for Mycobacterium organisms. ${ }^{6}$ Automated microbiologic identification systems do not differentiate well between Corynebacterium and $R$. equi, and this also may cause misdiagnosis. In our cases, "Corynebacterium” was identified in an automatic hemoculture system from 3 separate samples in 1 patient and in 5 samples in another. The clinician should be cautious before considering this agent to be a contaminant, particularly when the same agent is isolated from separate samples. Communication between the primary clinician who suspects $R$. equi infection and the laboratory could ensure accurate diagnosis and improve survival due to early treatment, avoiding secondary abscess. $^{6}$

The patient in case 1 was treated with vancomycin and rifampin for 6 weeks, but bacteremia persisted. $R$. equi is susceptible to vancomycin and rifampicin, although some reports have demonstrated an increase in rifampin resistance associated with the rpoB gene. ${ }^{7}$ Although adequate serum levels of vancomycin were detected, it should be noted that some studies have shown that the vancomycin does not reach an adequate concentration in the lung when serum levels are judged to be adequate. The antibiotic therapy in case 1 consisted of generic vancomycin. Conti Jr demonstrated in 1987 that both drugs had laboratory equivalence; however, this has not been clinically verified. ${ }^{8}$ Although there is no standard antibiogram for $R$. equi by CLSI (Clinical Laboratory Standards Institute), an E-test with 3 consecutive measures was performed to rule out the possibility of vancomycin resistance. ${ }^{9}$ The probability of endocarditis was low because there was no evidence of veg- etation in the transesophageal echocardiogram. Abscesses could maintain a continuous status of bacteremia, but there was no improvement in the clinical condition, and death was the outcome. In numerous situations, including cases of cerebral abscess, successful treatment starts with surgical resection, which is a consensus approach for Nocardia abscess. ${ }^{10-12}$ Another concern would be the capacity of penetration of vancomycin into abscesses. A previous study measured the concentration of vancomycin in a cerebral abscess, and the levels were similar to those of serum. ${ }^{13}$ This is virtually the first case of persistent $R$. equi bacteremia described. The possibility of resistance could be discharged, but the importance of standardization of susceptibility tests must be emphasized. A continuous maintenance of bacteremia due to lung and cerebral abscess would be the only explanation for this case.

In the case 2 , the bacteremia was successfully treated with imipenem and rifampin, but the patient died due to nosocomial infection after the surgical treatment of the abscess. Malacoplakia was related to $R$. equi infection in previous report. It is an unusual inflammatory condition characterized by the accumulation of macrophages with diagnostic Michaelis-Gutmann bodies. ${ }^{14}$ Although the pathogenesis of malacoplakia is unknown, it is thought to be an acquired disease secondary to immunosuppression and a possible defect in the oxygen-independent microbicidal system of macrophages. ${ }^{15}$

Infection caused by $R$. equi is a severe illness, given that more than one third of the patients die due to causes attributable to the infection, ${ }^{16}$ with an overall mortality of nearly $60 \% .^{1}$ A standard antibiogram method is necessary due the increase in resistance of gram-positive organisms. Surgical drainage of a $R$. equi abscess must be considered, and prospective studies analyzing the ideal approach should be done. Correct diagnosis and reporting of these cases are important for understanding of and increasing the experience with this rare infectious agent.

\section{ACKNOWLEDGEMENTS}

We thank Juliana Mori for the photography and its arrangement.

We thank for Denise Andreazzi for microbiological assistance and Hermes Higashino for clinical assistance.

\section{REFERENCES}

1. Kedlaya I, Ing MB, Wong SS. Rhodococcus equi infections in immunocompetent hosts: case report and review. Clin Infect Dis. 2001;32:39-46
2. Verville TD, Huycke MM, Greenfield RA, Fine DP, Kuhls TL, Slater LN. Rhodococcus equi infections of humans. Medicine. 1994;73:11932. 
3. Walsh RD, Schoch PE, Cunha BA. Rhodococcus. Infect Control Hosp Epidemiol. 1993;14:2827.

4. Votava M, Skalka B, Hrstkova H, Tejkalova R, Dvorska L. Review of 105 cases of isolation of Rhodococcus equi in humans. Cas Lek Cesk. 1997;22;136:51-3.

5. Golub B, Falk G, Spink WW. Lung abscess due to Corynebacterium equi. Report of first human infection. Ann Intern Med. 1967;66:11747

6. Funke G, von Graevenitz A, Clarridge JE 3rd, Bernard KA. Clinical microbiology of coryneform bacteria. Clin Microbiol Rev. 1997;10:12559 .

7. Asoh, N, Watanabe, H, Fines-Guyon M, Watanabe K, Oishi K, Kositsakulchai W, et al. Emergence of rifampin-resistant Rhodococcus equi with several types of mutations in the rpoB gene among AIDS patients in northern Thailand. J Clin Microbiol. 2003;41:2337-40.

8. Conte JE Jr. Comparative antibacterial activity of Vancocin and generic vancomycin. Antimicrob Agents Chemother. 1987;31:333-4.

9. Tomlin P, Sand C, Rennie RP. Evaluation of E test, disk diffusion and broth microdilution to establish tentative quality control limits and review susceptibility breakpoints for two aerobic actinomycetes. Diagn Microbiol Infect Dis. 2001;40:179-86.
10. Corne P, Rajeebally I, Jonquet O. Rhodococcus equi brain abscess in an immunocompetent patient. Scand J Infect Dis. 2002;34:300-2.

11. Kohl O, Tillmanns HH. Cerebral infection with Rhodococcus equi in a heart transplant recipient. J Heart Lung Transplant. 2002;21:1147-9.

12. Nasser AA, Bizri AR. Chronic scalp wound infection due to Rhodococcus equi in an immunocompetent patient. J Infect 2001; 42:6778 .

13. Levy RM, Gutin PH, Baskin DS, Pons VG. Vancomycin penetration of a brain abscess: case report and review of the literature. Neurosurgery. 1986;18:632-6.

14. Guerrero MF, Ramos JM, Renedo G, Gadea I, Alix A. Pulmonary malacoplakia associated with Rhodococcus equi infection in patients with AIDS: case report and review. Clin Infect Dis. 1999;28:1334-6.

15. Shin MS, Cooper JA Jr, Ho KJ. Pulmonary malacoplakia associated with Rhodococcus equi infection in a patient with AIDS. Chest. 1999;115:889-92.

16. Torres-Tortosa M, Arrizabalaga J, Villanueva JL, Galvez J, Leyes M, Valencia ME, et al. Prognosis and clinical evaluation of infection caused by Rhodococcus equi in HIV-infected patients: a multicenter study of 67 cases. Chest. 2003;123:1970-6. 